\title{
【Technical Note】 \\ Numerical study on the load direction effect on the performance of tilting pad-journal gas bearing
}

\author{
Tianwei LAI*, Yu HOU*, Lu NIU*, Yueqing ZHENG**** and Shuangtao CHEN* \\ *State Key Laboratory of Multiphase Flow in Power Engineering, Xi'an Jiaotong University, Xi'an 710049, P. R. China \\ **Institute of Mechanical Manufacturing Technology, China Academy of Engineering Physics, Mianyang 621900, P. R. China \\ E-mail: stchen.xjtu@mail.xjtu.edu.cn
}

Received 19 November 2013

\begin{abstract}
This paper quantitatively presents the effect of load directions on the static performance of gas titling pad-journal bearing (TPJB). The gas film thickness is determined in a subsystem composed of the rotor and one pad. Based on pressure distributions obtained from Reynolds equations, integration method is used to predict the bearing static performance. Pad forces and moment balances are considered in calculating the swing angles. Local and global parameters with different load directions are discussed analytically. With the variation of load direction, the global parameters of the bearing keep relatively stable, while the local parameters can make active changes.
\end{abstract}

Key words : Gas lubrication, Gas journal bearing, Tilting pad journal bearing (TPJB), Static performance, Load direction

\section{Introduction}

A tilting pad journal bearing (TPJB) has three or more pads which can adapt to load variations by adjusting their swing angles. Compared with fixed structure bearings, the pad rotation and translation together with preload are incorporated in the TPJB. Tilting pad journal bearings are often used in high speed and cryogenic machineries due to the inherent stability (Chen, et al., 1992; Gosh, et al., 2010; Gosh, et al., 2012; Kummer, et al., 2009). However, accidental uncertainties like pad fluttering and spragging (rotor and pad crashing) may occur in such complex structures (Pennacchi, et al., 2010; Yang, et al., 2009; Hargreaves and Fillon, 2007). In order to improve bearing performance in high speed turbomachinery, Andres used the hybrid flexible structure (Andres, 2006). Ertas integrated compliant hybrid bearing with flexibly mounted wire mesh dampers to maximize the load capacity and effective damping ( Ertas, 2009).

The schematic diagram of three-pad TPJB is shown in Figure 1. According to load directions, there are two main configurations, load between pads (LBP) (Figure 1 (a)) and load on pad (LOP) (Figure 1 (b)). Childs experimentally determined the static performance of oil bearing with LBP configuration. As for LOP style, Trip measured the steady state eccentricities (Tripp and Murphy, 1984), while Andres measured the bearing displacement and pad edge temperatures (Andres and Jackson, 1998). DeCamillo compared the oil temperature profiles between the two configurations (Decamillo and Brockwell, 2001). For both structures, Wygant measured the oil film thickness and attitude angles with different preload ratios (Wygant, 2004). Jones showed the effect of load direction on the steady state behavior, especially on the minimal oil film thickness from one "between pad" position to another (Jones and Martin, 1979). The oil viscosity is about 1000 times larger than gas, therefore, the fluid temperature change in gas lubricated TPJB will be negligible. General characteristics of the TPJB will be discussed regardless of types of the lubricant. 


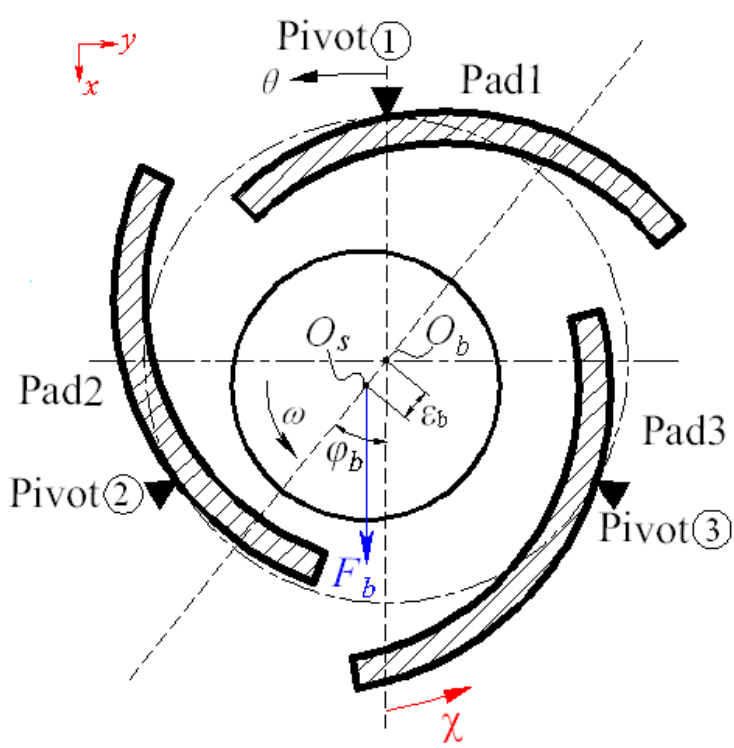

(a)
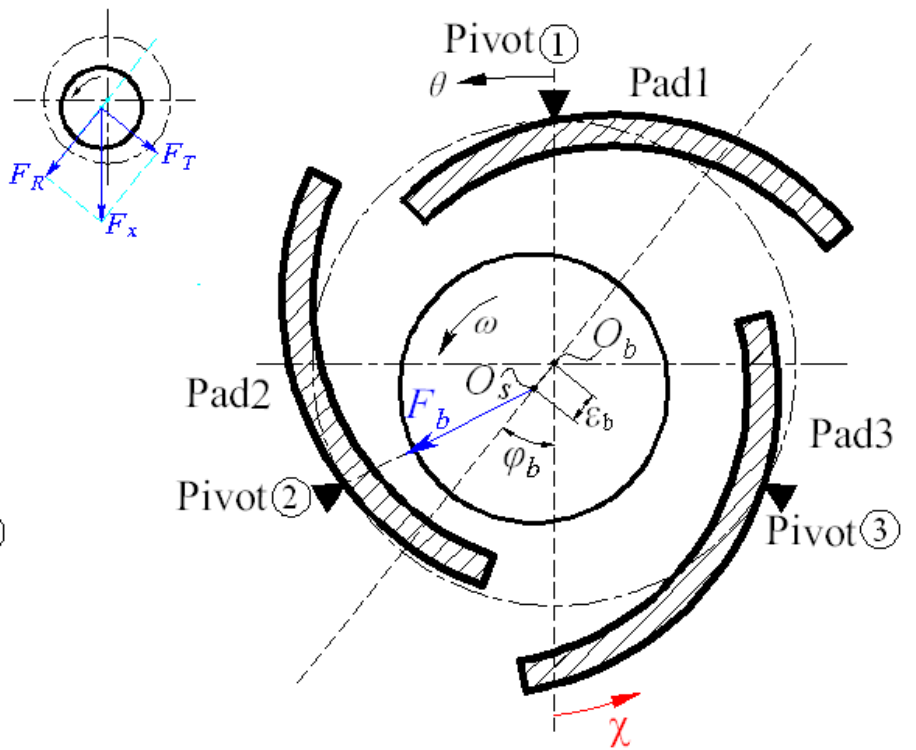

(b)

Figure 1.TPJB a) LBP b) LOP

In rotor bearing system, the rotor and one of the pads form a subsystem in which the local parameters such as local attitude angle and local eccentricity determine the gas film thickness and pressure distribution. Fu mathematically analyzed the clearance space by identifying the three distinct stages of journal position and the pad slide circumferentially around the bearing housing which applies to some special constitution (Fu and Parkins, 1995). Combining the single bearing pad load capacity and friction, Castelli and Gunter described the finite difference solution of the self acting gas lubricated journal bearing for static performance (Castelli, et al., 1964; Gunter, et al., 1963; Gunter, et al.,1964). Elwell presented a general guide for bearing design computation (Elwell and Findlay, 1969).

While most of the study on the TPJB is focused on the oil lubricant, the TPJB using gas is of interest for high-speed applications including the cryogenic turbo-expander. In those high-speed applications, the load direction may vary with the change in operation conditions, which is critical for the stability of operation. In this paper, we perform the theoretical study on the gas TPJB, and investigate the load direction effect on the tilting pad journal bearing in detail. When the load direction transits from one of the bottom pads to another, the local and global parameters of the bearing make adjustments in order to adapt to the change. The characteristics of the local parameters will be of great importance for the stable operation of the bearing.

\section{Nomenclature}

$\begin{array}{cl}C_{b} & \text { nominal radial clearance between pad and shaft }\left(=R_{\mathrm{p}}-R_{\mathrm{s}}\right), \mathrm{mm} \\ C_{v} & \text { Pivot } \sim \text { circle radius clearance }\left(=R_{v}-R_{s}\right), \mathrm{mm} \\ F^{i} & \text { gas film force on } i^{\text {th }} \text { pad, } \mathrm{N} \\ F_{b} & \text { bearing load, } \mathrm{N} \\ h & \text { gas film thickness, mm } \\ H & \text { dimensionless gas film thickness }\left(=h / C_{\mathrm{b}}\right) \\ L & \text { bearing length, } \mathrm{mm} \\ m & \text { preload ratio }\left(=1-C_{v} / C_{b}\right) \\ M & \text { pad moment about pivot, } \mathrm{N} \cdot \mathrm{m} \\ O_{\mathrm{s}} & \text { shaft center } \\ O_{b} & \text { bearing center } \\ O_{p}{ }^{i} & \text { original pad center } \\ O_{p}{ }^{{ }_{1}} & \text { pad centers with swing angles } \\ p & \text { gas film pressure, } \mathrm{Pa} \\ P & \text { dimensionless gas film pressure }\left(=p / p_{a}\right) \\ p_{a} & \text { ambient pressure, } \mathrm{Pa} \\ r_{\xi} & \text { radial distance between force direction and pivot }\end{array}$




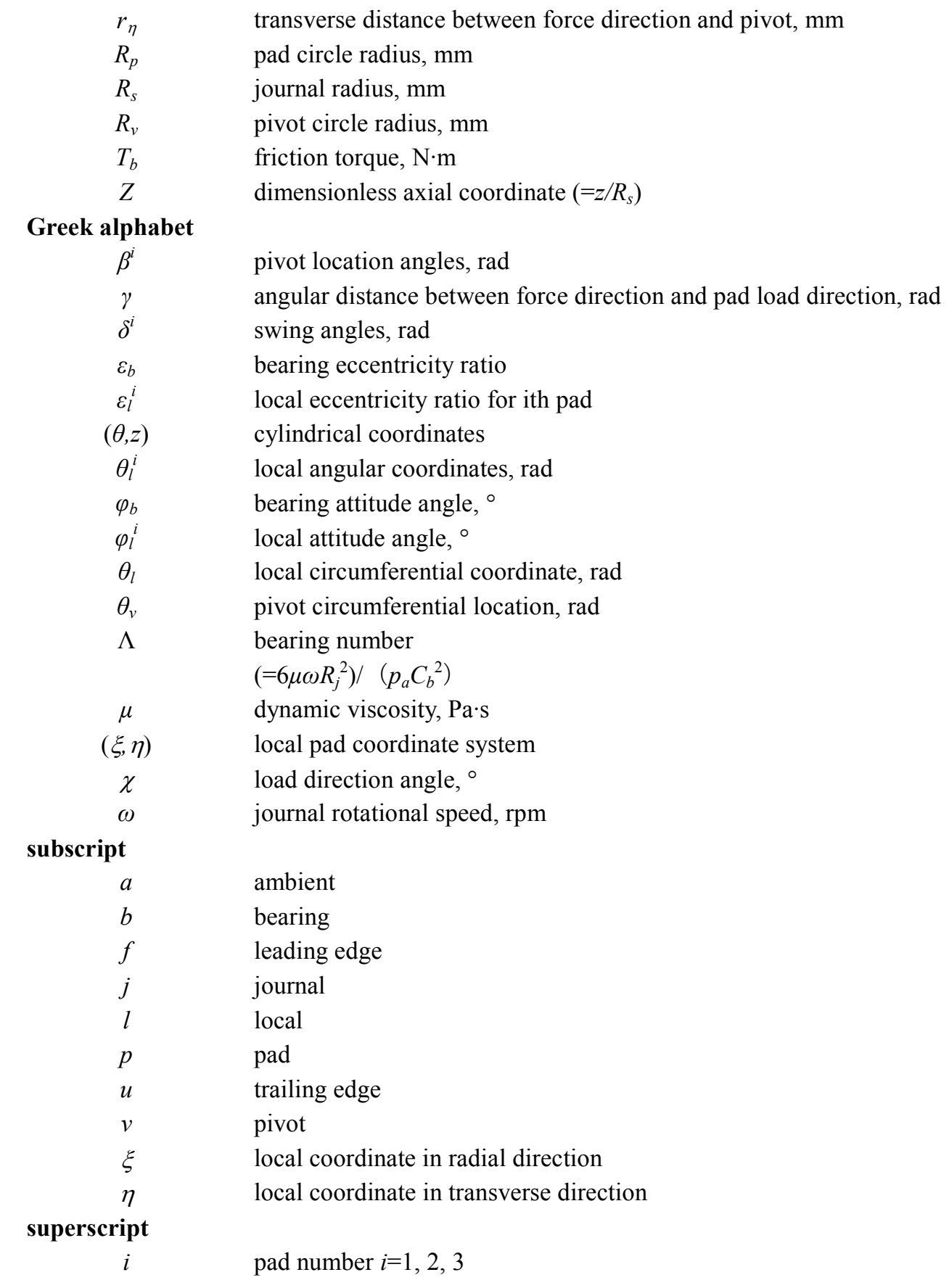

\section{Numerical model of TPJB}

In this study, three pad-journal gas bearing is chosen as a specific example shown in Figure 1. According to the isothermal condition and laminar flow pattern in the bearing, the following assumptions can be made to simplify the physical model (Szeri, 2011).

1) Gas viscosity is constant;

2) Bearing misalignment is negligible;

3) Pad thickness and mass are negligible;

4) Fluid inertia is negligible.

Based on the above assumptions and the coordinate system in Figure 1 (a), the fluid film pressure distribution on the pads can be described by the following Reynolds equation: 


$$
\frac{\partial}{\partial \theta}\left(p h^{3} \frac{\partial p}{\partial \theta}\right)+\frac{\partial}{\partial z}\left(p h^{3} \frac{\partial p}{\partial z}\right)=6 \mu \omega R_{s} \frac{\partial(p h)}{\partial \theta}+12 \mu \frac{\partial(p h)}{\partial t}
$$

We rewrite Equation (1) in the dimensionless form in steady state:

$$
\frac{\partial}{\partial \theta}\left(P H^{3} \frac{\partial P}{\partial \theta}\right)+\frac{\partial}{\partial \mathrm{Z}}\left(P H^{3} \frac{\partial P}{\partial \mathrm{Z}}\right)=\Lambda \frac{\partial}{\partial \theta}(P H)
$$

The corresponding dimensionless boundary conditions are:

$$
\left\{\begin{array}{l}
P(\theta, Z=0)=1.0 \\
P\left(\theta, Z=L / R_{s}\right)=1.0 \\
P\left(\theta=\theta_{0}, Z\right)=1.0 \\
P\left(\theta=\theta_{1}, Z\right)=1.0
\end{array}\right.
$$

Finite element formulations of governing equations are obtained using Galerkin method the same as in reference (Hou, et al., 2011).

Instead of using the gas film expression directly in reference (Andres, 2006), the gas film is determined by relative geometrical relations of subsystem such as local eccentricity and attitude angle as shown in Figure 2(a). Three main structure parameters, preload ratio $m$, global eccentricity ratio $\varepsilon$, and pad swing angle $\delta^{i}$ are incorporated into film thickness calculation with counterclockwise direction. Pad radial and transverse displacements are neglected by setting the pivot as stiff support. Vectors denotation of one pad system is presented in Figure 2 (b). $a_{2}{ }^{i}$ is almost perpendicular to $a_{3}{ }^{i}$ due to the large ratio of $R_{j} / C_{b}$. The dimensionless gas film thickness along circumferential direction for each pad can be represented using local eccentricity $\varepsilon^{i}$ and local angular coordinates $\theta_{l}^{i}$ as follows.

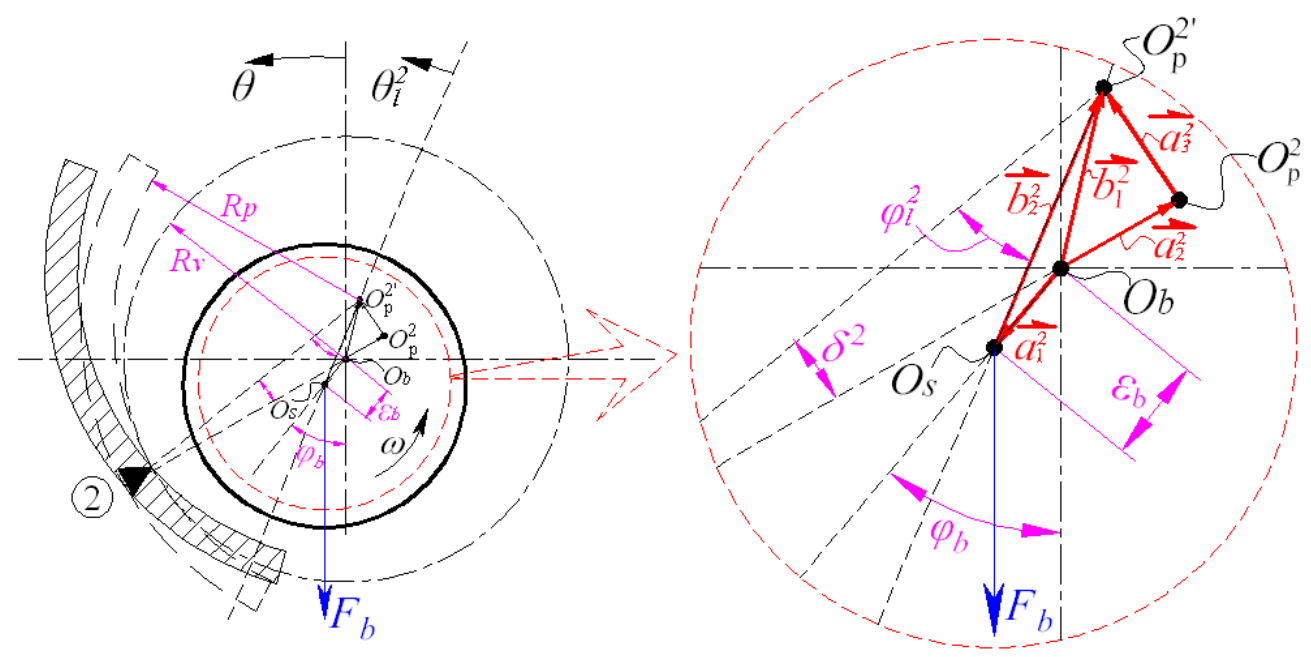

Figure 2 Local subsystem and vector method

$$
H^{i}=1-\varepsilon^{i} \cos \theta_{l}^{i}
$$

where

$$
\varepsilon^{i}=\left|\overrightarrow{b_{2}^{i}}\right|=\left|\overrightarrow{b_{1}^{i}}-\overrightarrow{a_{1}^{i}}\right|=\left|\overrightarrow{a_{2}^{i}}+\overrightarrow{a_{3}^{i}}-\overrightarrow{a_{1}^{i}}\right|
$$




$$
\begin{aligned}
& \theta_{l}^{i}=\theta^{i}-\theta_{b 2}{ }^{i} \\
& \overrightarrow{a_{1}^{i}}=\varepsilon(\cos (\varphi)+i \sin (\varphi)) \\
& \overrightarrow{a_{2}^{i}}=m\left(\cos \left(\theta_{k}^{i}-\pi\right)+i \sin \left(\theta_{k}^{i}-\pi\right)\right) \\
& \overrightarrow{a_{3}^{i}}=\delta^{i}\left(\cos \left(\theta_{k}^{i}-\pi / 2\right)+i \sin \left(\theta_{k}^{i}-\pi / 2\right)\right) \\
& \overrightarrow{b_{1}^{i}}=\overrightarrow{a_{2}^{i}}+\overrightarrow{a_{3}^{i}} \\
& \overrightarrow{b_{2}^{i}}=\overrightarrow{b_{1}^{i}}-\overrightarrow{a_{1}^{i}}=\left|b_{2}{ }^{i}\right|\left(\cos \left(\theta_{b_{2}}{ }^{i}\right)+i \sin \left(\theta_{b_{2}}{ }^{i}\right)\right)
\end{aligned}
$$

The gas film force acting on the pad is in balance with the reacting force of the pivot by neglecting pad mass. The pads will adjust their swing angles for the moment balance with respect to pivot center. The fluid film differential moment about the pad pivot is found by taking the cross product of vector $\vec{r}$ with the differential force vector $d \vec{F}$, i.e.

$$
d \vec{M}=\vec{r} \times d \vec{F}
$$

Neglecting the pad thickness, the vector $\vec{r}$ in Figure 3 can be determined by

$$
\vec{r}=\left[\left(R_{s}(1-\cos \gamma)\right) \vec{\xi}+\left(R_{s} \sin \gamma\right) \vec{\eta}\right]
$$

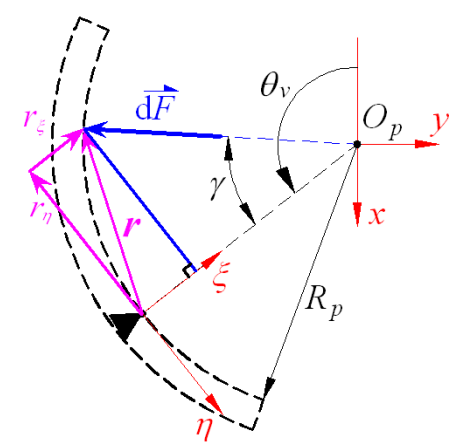

Figure 3 Pad moment balance

The differential fluid film force vector can be written as

$$
d \vec{F}=d F_{\xi} \vec{\xi}+d F_{\eta} \vec{\eta}
$$

where $d F_{\xi}=-\left(p-p_{a}\right) \cos \gamma R_{s} d \gamma d z$ and $d F_{\eta}=\left(p-p_{a}\right) \sin \gamma R_{s} d \gamma d z$.

The differential moment is therefore obtained by substituting Equation (14) into Equation (12)

$$
\begin{aligned}
& d \vec{M}=-\left(p-p_{a}\right) R_{s}^{2} \sin \gamma d \gamma d z \vec{\kappa} \\
& \vec{\kappa}=\vec{\xi} \times \vec{\eta} \\
& \iint_{\Omega} d \vec{M}=-\iint_{\Omega}\left(p-p_{a}\right) R_{s}^{2} \sin \gamma d \gamma d z \vec{\kappa}
\end{aligned}
$$


By surface integration on each pad, the net moment on the pad should be zero for non-rotational condition. With the calculation on each pad, the bearing performance can be predicted.

The bearing load:

$$
\begin{aligned}
& {\left[\begin{array}{l}
F_{X} \\
F_{Y}
\end{array}\right]=\int_{-L / D}^{L / D} \int_{0}^{2 \pi}\left[\begin{array}{l}
-p \cos \theta \\
-p \sin \theta
\end{array}\right] d \theta d Z} \\
& {\left[\begin{array}{l}
F_{R} \\
F_{T}
\end{array}\right]=\int_{-L / D}^{L / D} \int_{0}^{2 \pi}\left[\begin{array}{l}
-p \cos (\theta-\varphi) \\
-p \sin (\theta-\varphi)
\end{array}\right] d \theta d Z} \\
& F_{b}=\sqrt{F_{x}^{2}+F_{y}^{2}}=\sqrt{F_{R}^{2}+F_{T}^{2}}
\end{aligned}
$$

Attitude angle :

$$
\varphi=-\tan ^{-1}\left(\frac{F_{T}}{F_{R}}\right)
$$

Dimensionless friction torque:

$$
T_{f}=\frac{f_{t}}{p_{a} c R_{s}{ }^{2}}=\int_{-L / D}^{L / D} \int_{0}^{2 \pi}\left[\frac{H}{2} \frac{\partial P}{\partial \theta}+\frac{\Lambda}{6 H}\right] d \theta d Z
$$

After gas film determination, Newton-Raphson method is adopted in solving the non-linear matrix equations. Grid independence has been evaluated and $10 \times 10$ mesh (element number $\mathrm{NE}=100$ ) ensures that the maximal variation in loading capacity is within $0.5 \%$ when the node points doubled. Pressure distribution on each pad is obtained independently; thereafter, gas film hydrodynamic forces can be obtained by surface integration on each pad. Meanwhile, the pressure field will counter-act on the rotatable pad.

In the computing process, as shown in Figure 4 bearing eccentricity ratio is given and bearing attitude angle can be presumed and then determined by iteration. Pad rotational angle is assumed in the range of $\left(-\delta_{0},+\delta_{0}\right)$ and by-section method is used in angle determination. On condition that the fluid pressure moment on the pad with $-\delta_{0}$ and $+\delta_{0}$ are of the same sign, the searching extent is enlarged to be $\left(-1.2 \delta_{0},+1.2 \delta_{0}\right)$ until the pad rotational angle at which the pad moment is zero is being included. The moment balance will update the pad rotational angle until balance criteria is satisfied. In case that the gas film thickness became negative before an appropriate rotational angle can be determined, the program will stop. Meeting the force $\left(F_{Y}<10^{-3}\right)$ and moment equilibrium $\left(M_{p}<10^{-6}\right)$ criteria, the results will be outputted. There will be a risk that the assumed bearing attitude angle in the iteration may cause negative film thickness, namely calculation collapse.

\section{Results and discussions}

The static performance of the bearing with structure parameters in Table 1 is determined using the model in Chapter 2 under the variations of load direction in Table 2. Air is used as lubricant in the bearing. 


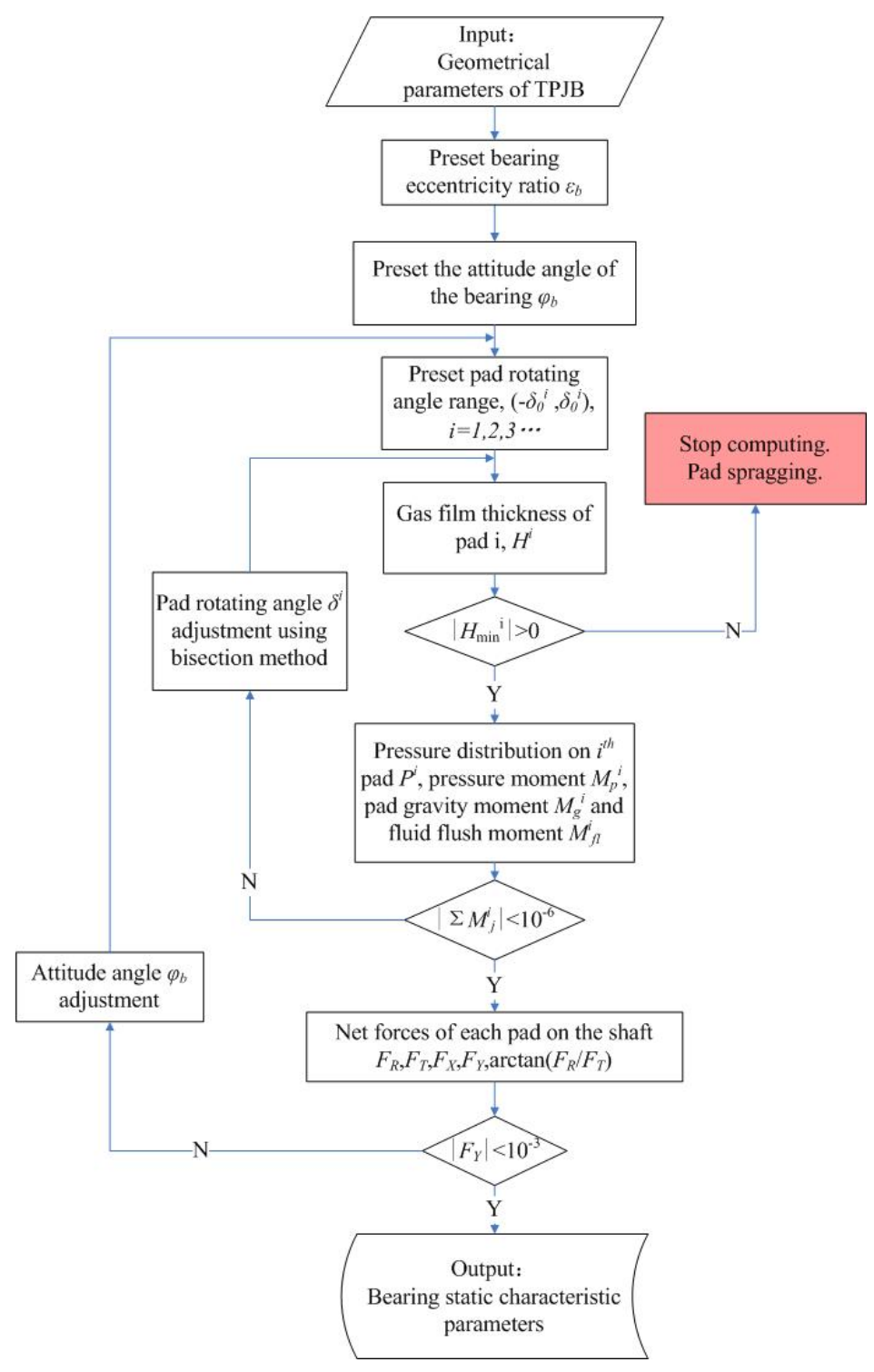

Figure 4 Computing algorithm of TPJB

Table 1 Geometric and operating conditions

\begin{tabular}{llll}
\hline Parameter & Value & Parameter & Value \\
\hline Bearing length $(\mathrm{mm})$ & 33 & Pad angular extent $\left(^{\circ}\right)$ & $94.5,94.5,94.5$ \\
Journal radius $(\mathrm{mm})$ & 20 & Pivot location ratio & 0.65 \\
$\mathrm{~m}$ & 0.376 & Pivot location in bearing $\left(^{\circ}\right)$ & $0,130,260$ \\
$\varepsilon_{\mathrm{b}}$ & 0.164 & Nominal radial clearance $C_{b}(\mu \mathrm{m})$ & 29.5 \\
Lubricant viscosity $(\mathrm{Pa} \cdot \mathrm{s})$ & $18.7 \times 10^{-6}$ & $\Lambda$ & 3.0 \\
\hline
\end{tabular}

\subsection{Response of local parameters to load direction}

Local eccentricity ratio reflects the approaching extent of the rotor to the pad, and it is determined by Equation 11 . In Figure 5 (a), local eccentricity ratios of the three pads are around 0.6. When the load direction shifts from pad 2 to 
pad 3 , the eccentricity ratio of pad 2 is decreasing from 0.62 to 0.59 , while that of pad 3 is increasing from 0.59 to 0.62 . For the unloaded pad 1, its eccentricity maintains stable around 0.60 . It can be noted that a smaller load requires less local eccentricity. Therefore, for the loaded pad 2 and pad 3, they need higher local eccentricities. When the load is located between the two pads, $\chi=0^{\circ}$, the maximal local eccentricity ratio of the three pads is 0.60 . This indicates that there will be more operation margins for load between pads mode.

Local attitude angle reflects the relative position between the rotor and pad, and it can also be determined by Equation11. The trends of local attitude angles with load direction are shown in Figure 5 (b). When the load direction angle $\chi$ changes from $-50^{\circ}$ to $50^{\circ}$, the local attitude angle of pad 1 increases from $56^{\circ}$ to $62^{\circ}$ first and decreases to $56^{\circ}$. At $\chi=0^{\circ}$, pad 1 has a maximal local attitude angle since the changing load direction is nearly symmetrical about the perpendicular line. In the whole process, the local attitude angle of pad 1 is relatively high compared to that of the other two pads due to the unloaded state. The local attitude angle of pad 2 and pad 3 goes in opposite direction when the load transits from pad 2 to pad 3. When the load direction angle $\chi$ changes from $-50^{\circ}$ to $50^{\circ}$, local attitude angle of pad 2 increases from $39^{\circ}$ to $55^{\circ}$, meanwhile the local attitude angle of pad 3 decreases from $55^{\circ}$ to $39^{\circ}$.

Pad swinging is one of the most significant characteristics of the tilting pad bearing. In Figure 5 (c), as the load direction varies, the three pads swing around their pivots in different ways. When load direction transits from pad 2 to pad 3, swing angle of pad 1 increase from $0.03^{\circ}$ to $0.05^{\circ}$ which means that pad 1 swings in counterclockwise direction. Meanwhile, swing angle of pad 2 firstly swings clockwise and then in the opposite direction around $0.03^{\circ}$. Swing angle of pad 3 swings clockwise from $0.05^{\circ}$ to $0.03^{\circ}$. It should be noted that the swing angles of the three pads are asymmetrical about the perpendicular line which can be attributed to the intrinsic nonsymmetrical structure of the bearing.

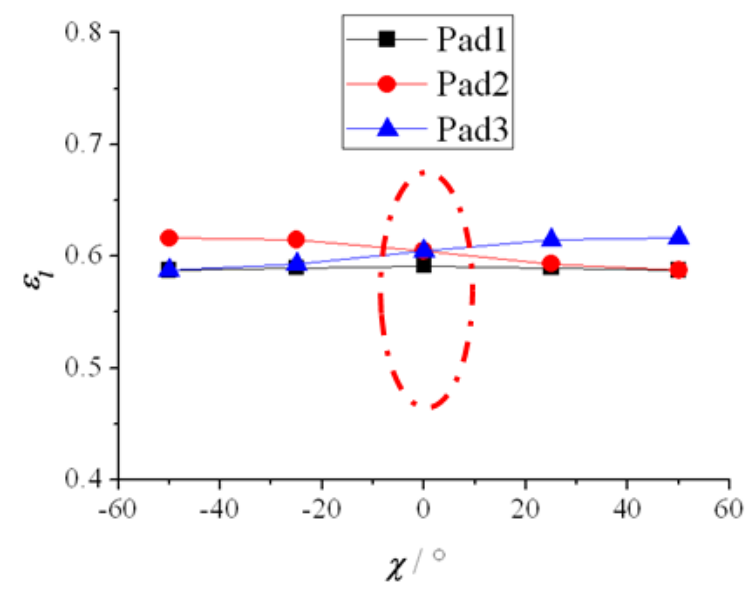

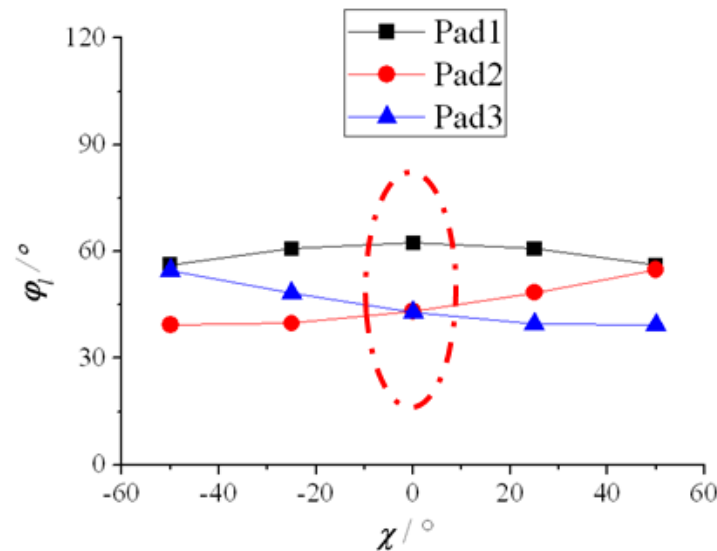

(b)

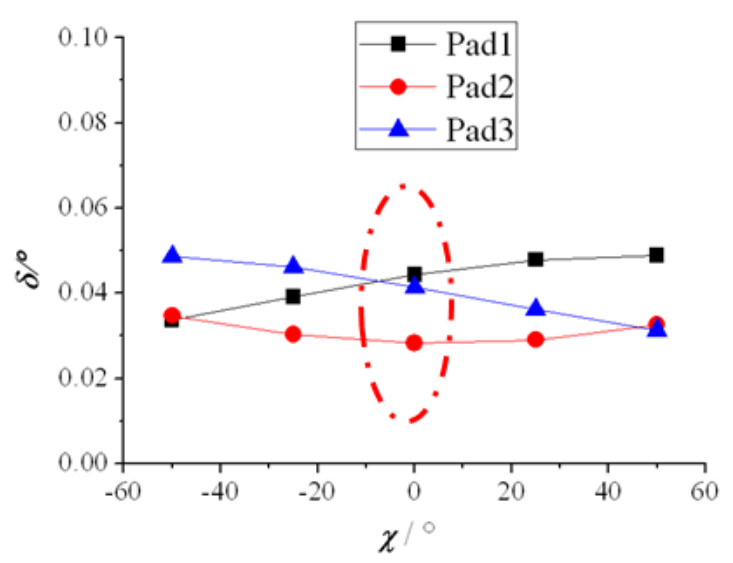

(c)

Figure 5 Local parameters (a) local eccentricity; (b) local attitude angle; (c) pad swing angle

Using the above local parameters in each subsystem, gas film thickness can be determined by Equation 4 similar to plain journal bearing. In Figure 6 (a), the dimensionless gas film thicknesses at the three pads are arranged in 
circumferential direction. When load direction transits from pad 2 to pad 3, the gas film thickness increases on pad 2 and decreases on pad 3. The minimal gas film thickness on pad 2 increases from 0.39 to 0.46 . The minimal gas film thickness on pad 3 decreases from 0.46 to 0.39 . The gas film thickness variation is small on pad 1 due to the nearly unloaded state. As long as the gas film thickness in the bearing is determined, the dimensionless pressure field can be obtained by solving the Reynolds equation. For convenience of comparison, the pressure field at the centerline of the journal bearing is shown in Figure 6 (b). It should be noted that the maximal pressure on pad 2 decreases from 1.9 to 1.67, while the maximal pressure on pad 3 increases from 1.67 to 1.9. The maximal pressure on pad 1 maintains around 1.53 .

Figure 7 (a) to (e) show the variations of pressure field as the load direction moves from pad 2 to pad 3 . With the change of load direction, the maximal pressure on pad 2 decreases and the maximal pressure on pad 3 increases. In the whole process, the pressure on pad 1 remains relatively stable.

After the pressure field of the bearing is obtained, the acting force on the pad can be derived by surface integration. With the load direction shifted from pad 2 to pad 3, as Figure 8 shows, the load on pad 2 is decreasing from $30.6 \mathrm{~N}$ to 16.1 $\mathrm{N}$ and the load on pad 3 is increasing from $16.1 \mathrm{~N}$ to $30.6 \mathrm{~N}$. When the load direction is perpendicular, namely $\chi=0^{\circ}$, the loads on pad 2 and pad 3 are almost the same which are around $25.6 \mathrm{~N}$. The load capacity of pad 1 keeps relative stable around $14 \mathrm{~N}$.

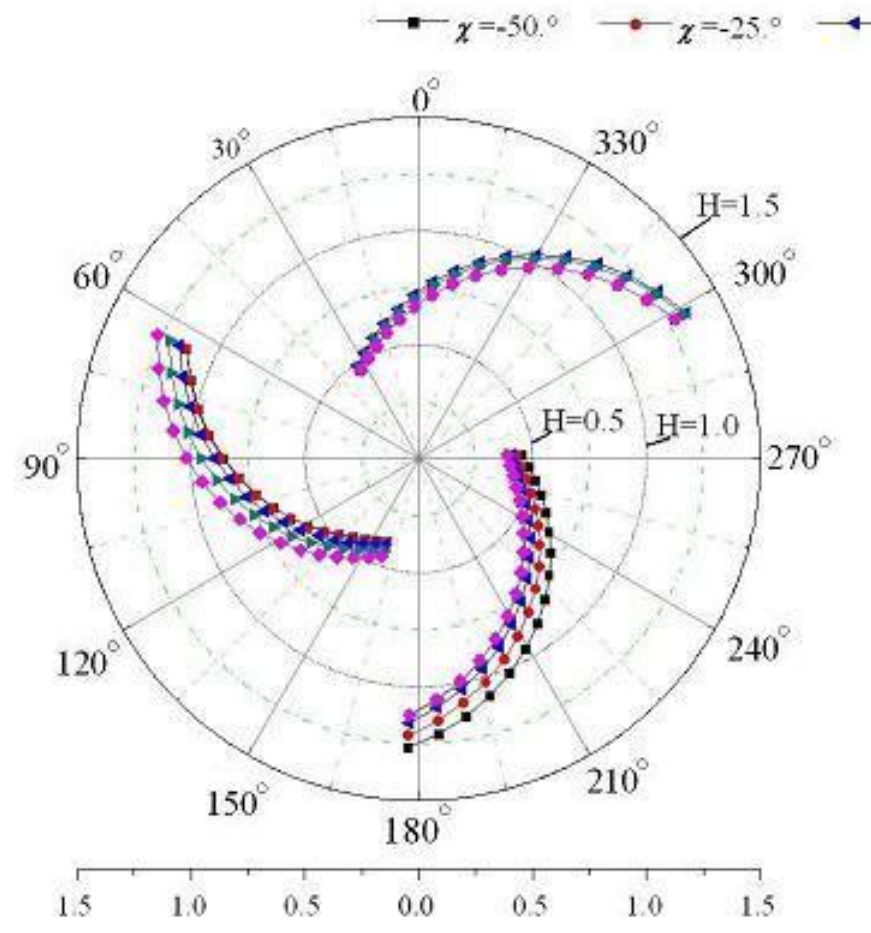

(a)

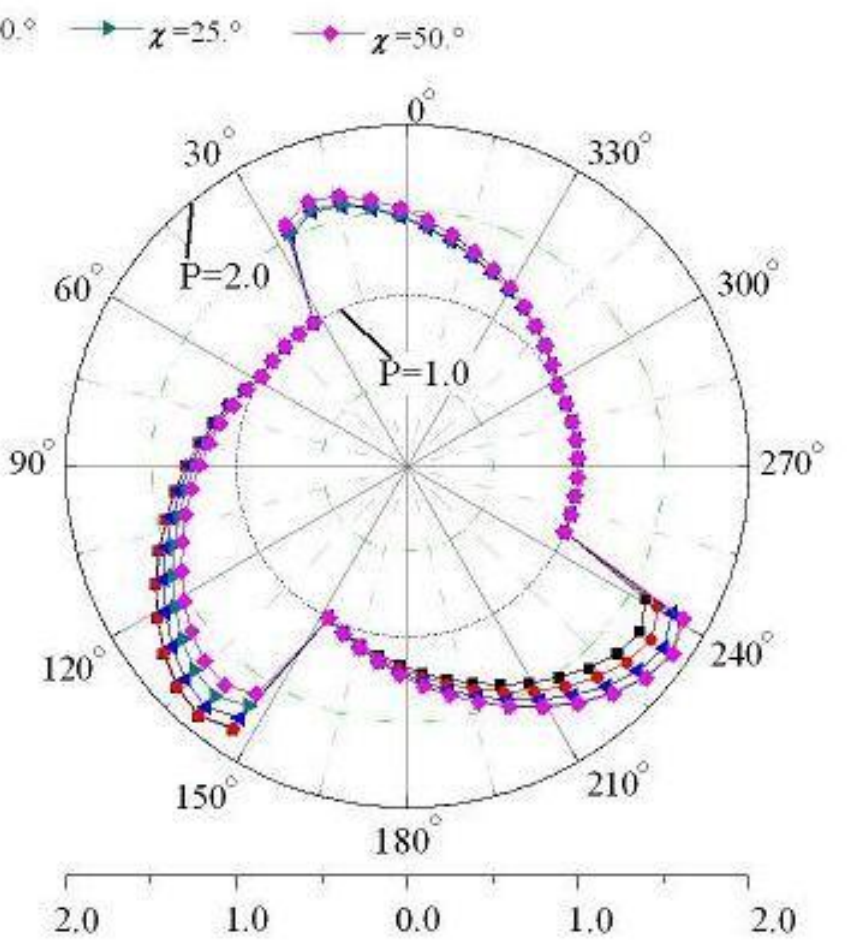

(b)

Figure 6 Circumferential (a) gas film thickness; (b) dimensionless pressure field 


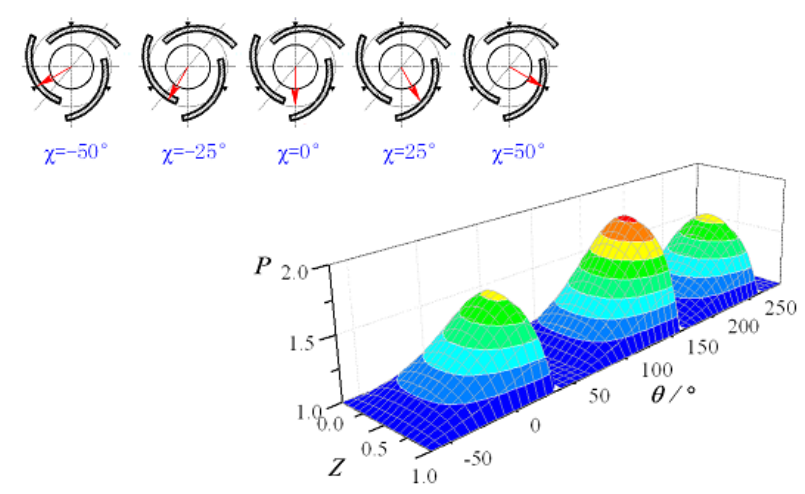

(a)

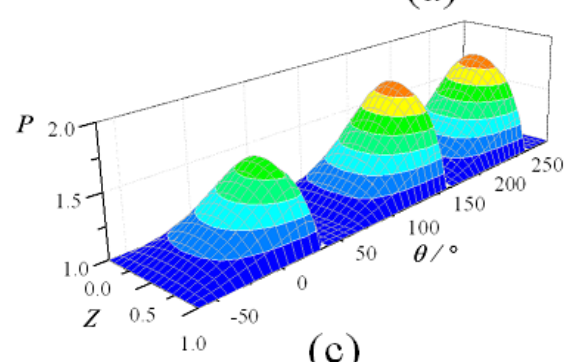

(c)

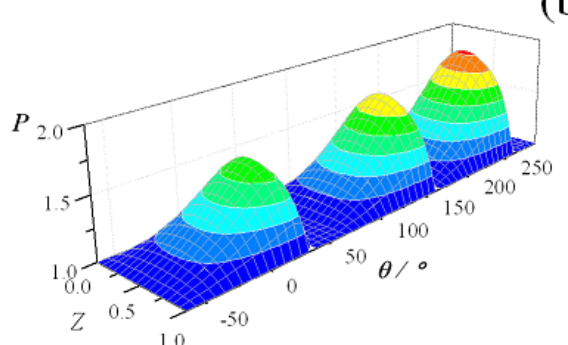

(d)

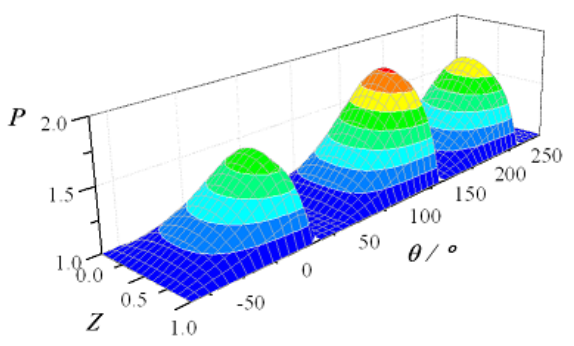

(b)

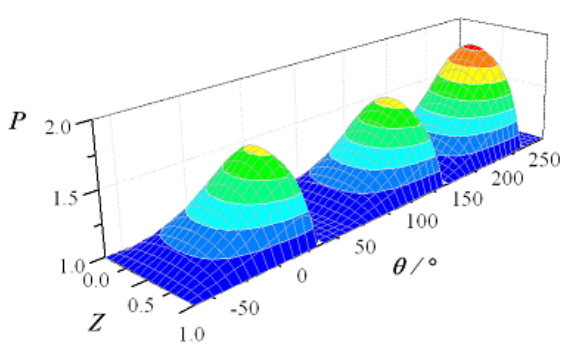

(e)

Figure 7 Pressure field in bearing (a) $\chi=-50^{\circ}$; (b) $\chi=-25^{\circ}$; (c) $\chi=0^{\circ}$; (d) $\chi=25^{\circ}$; (e) $\chi=50^{\circ}$

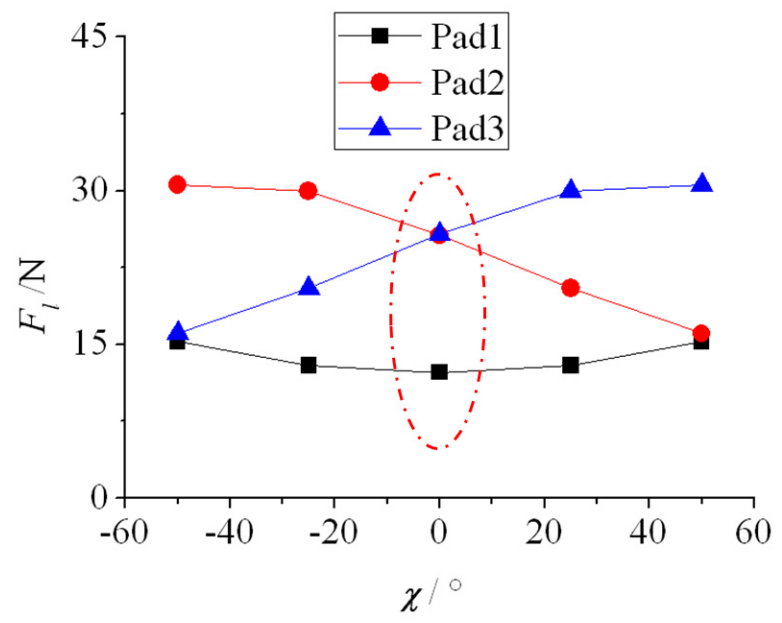

Figure 8 Pad loads

\subsection{Response of global parameters to load direction}

The bearing characteristics can be determined by the subsystem performance. The global parameters of the bearing are obtained by Equation 18 to Equation 22 and shown in Figure 9, which include attitude angle, load capacity, and friction torque. Bearing attitude angle maintains small values from $-10^{\circ}$ to $10^{\circ}$ with the variation of load direction, which is consistent with the experimental results from the references (Wygant et al., 2004; Jones and Marin, 1979). This can be attributed to the actively swinging characteristics of the pads. The attitude angle of the bearing is closely correlated to the load direction. Besides, given the same eccentricity ratio, LBP configuration has higher bearing load than LOP configuration. Under the same operation parameters, the bearing loads are $16 \mathrm{~N}$ and $14.8 \mathrm{~N}$ when the load directions are towards pad 2 and on pad 3 respectively, which can be attributed to the unsymmetrical configuration of 
the bearing about the $\chi=0^{\circ}$ loading line. The maximal load is $19.2 \mathrm{~N}$ with load between pad 2 and 3 . The maximal load difference is about $23 \%$ between LOP configuration with load on pad 3 and LBP configuration with load between pad 2 and 3. The net force from the two loaded pads and one almost unloaded pad in LBP is greater than that in LOP whose load is carried by only one pad and the other two pads maintain almost unloaded. However, the friction torque, which results from the shearing forces tangential to the pads, changes little with the load direction around $0.06 \mathrm{~N} \cdot \mathrm{m}$. Comparatively speaking, the friction torque with LBP configuration is a little bigger.

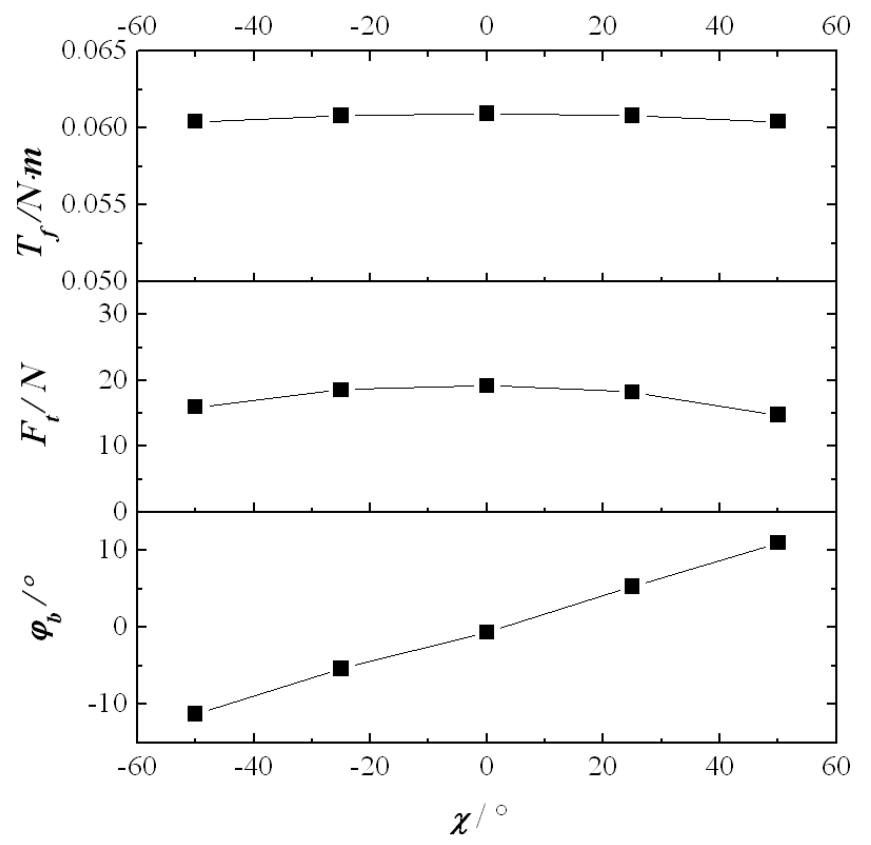

Figure 9 Global parameters

\section{Conclusions}

In TPJB, pad swing angle and local eccentricities make adjustments in response to the changes of load direction with a given configuration and operation condition. This paper presents the relationship between the loading directions and the static performance of TPJB with emphasis on subsystem parameters. With the load direction variations, the following conclusions can be made:

1. The local parameters such as pad swing angles, local eccentricity and local attitude angles can respond to the variations of load direction actively; The maximal local eccentricity and attitude angle for LBP configuration is smaller than that of the LOP configuration, which indicates that the LBP configuration possess more operation safety margin;

2. At the same bearing eccentricity ratio, the bearing load of gas lubricated TPJB with LBP configuration is about $23 \%$ bigger than the LOP configuration. The bearing load can be adaptive to the variation of load direction by adjusting the pad pitch angle without the journal derailing from its stable position. The friction torque of the bearing maintains relative stable.

3. Three pad-journal gas bearing has been chosen as a typical configuration. The conclusions arrived here can be apply to the TPJB with more than three pads.

\section{Acknowledgement}

This project was supported by National Nature Science Foundation of China (NSAF 11176023, NSFC 51306135) and the National Basic Research Program of China (2011CB706505). 


\section{References}

Andres, L. S., Hybrid flexure pivot-tilting pad gas bearings: analysis and experimental validation, ASME Journal of Tribology, Vol.128 (2006), pp.551-558.s

Andres, L. S. and Jackson, M. C., Measurements of the static load (on pad) performance and pad temperatures in a flexure-pivot tilting-pad bearing, Tribology Transactions, Vol.41 (1998), pp.225-232.

Castelli, V. C., Stevenson, C. H. and Gunter, JREJ., Steady-state characteristics of gas-lubricated, self-acting, partial-arc journal bearings of finite width, ASLE Transactions, Vol.7 (1964), pp. 153-167.

Chen, C. Z., Dong, Z. H., Cui, G. N., et al. Experimental study of three-titling-pad self-acting journal gas bearing expansion turbine, Cryogenics, Vol.32( Supplement 1) (1992), pp.76-79.

Childs, D. and Harris, J., Static performance characteristics and rotordynamic coefficients for a four-pad ball-in-socket tilting pad journal bearing, ASME Conference Proceedings, 43154 ( 2008), pp.861-871.

Decamillo, S. M. and Brockwell, K., A study of parameters that affect pivoted shoe journal bearing performance in high-speed turbomachinery, In: Proceedings of the 30th turbomachinery symposium, Houston, Texas, 18-20 September 2001, pp. 9-22.

Elwell, R. C. and Findlay, J. A., Design of pivoted pad journal bearings, Journal of Lubrication Technology Series F.-Trans ASME, Vol.91 (1969), pp. 87-103.

Ertas, B. H., Compliant hybrid journal bearings using integral wire mesh dampers, Journal of Engineering for Gas Turbines and Power 2009, Vol.131, paper no. 022503, pp.1-11.

Ghosh, S., Mukherjee, P. and Sarangi, S., Development of bearings for a small high speed cryogenic turboexpander, Industrial Lubricication and Tribology, Vol.64 (2012), pp.3-10.

Ghosh, S. K., Sahoo, R. K. and Sarangi, S. K., Experimental performance study of cryogenic turboexpander by using aerodynamic thrust bearing, Applied Thermal Engineering, Vol.30 (2010), pp.1304-1311.

Gunter, J. R. E. J., Hinkle, J. G. and Fuller, D. D., The effects of speed, load, and film thickness on the performance of gas-lubricated, tilting-pad journal bearings, ASLE Transactions, Vol.7 (1964), pp.353-365.

Gunter, J. R. E. J., Hinkle, J. G., and Fuller. D. D., Theoretical and experimental investigation on gas-lubriated, pivoted-pad journal bearings, ASLE Transactions, Vol.6 (1963), pp.346-357.

Hargreaves, D. J. and Fillon, M., Analysis of a tilting pad journal bearing to avoid pad fluttering, Tribology International, Vol.40 (2007), pp.607-612.

Hou, Y., Chen, S. T., Chen, R. G., et al. Numerical study on foil journal bearings with protuberant foil structure, Tribology International, Vol.44(2011), pp.1061-1070.

Jones, G. J. and Martin, F. A. Geometry-effects in tilting-pad journal bearings, ASLE Trans, Vol.22 (1979), pp.227-244.

$\mathrm{Fu}$, W. B. and Parkins, D.W., Mathematical-analysis for the clearance space of a tilting-pad journal bearing, STLE Tribology Transactions, Vol.38 (1995), pp.525-532.

Kumar, S., Ranjan, R. and Ghosh, S. K., Design of pivot less tilting pad journal bearing for cryogenic turboexpander, In: 14th national conference on machines and mechanisms, Durgapur, India, 17-18 December 2009, pp. 380-385.

Pennacchi, P., Vania, A., Chatterton, S., et al., Case history of pad fluttering in a tilting-pad journal bearing, In: Proceedings of the ASME Turbomachinery Exposition, Glasgow, 14-18 June 2010, pp. 1-7.

Szeri, A. Z., Fluid film lubrication, 2nd ed. New York: Cambridge University Press, 2011, p. 454.

Tripp, H. and Murphy, B., Eccentricity measurements on a tilting pad bearing, ASLE Transactions, Vol.28 (1984), pp.217-224.

Wygant, K. D., Flack, R. D. and Barrett, L. E., Measured performance of tilting-pad journal bearings over a range of preloads - part I: static operating conditions, Tribology Transactions, Vol.47 (2004), pp.576-584.

Yang, S., Kim, C. and Lee, W., Prevention of fluttering fatigue damage in a tilting pad journal bearing, Tribology International, Vol.42 (2009), pp.816-822. 